\section{Adaptación y validación de la versión chilena del cuestionario "Evaluación de la Atención de Enfermedades Crónicas para pacientes"}

\author{
MARÍA ELENA LAGOS G. ${ }^{1, \mathrm{a}, \mathrm{d}}$, ALIDE SALAZAR M., ${ }^{1, \mathrm{~b}, \mathrm{e}}$ \\ PATRICIO SALAS F.,
}

\section{Assessment of a Chilean Spanish version of the Patient Assessment of Chronic Illness Care Questionnaire}

Background: The Patient Assessment of Chronic Illness Care (PACIC) questionnaire is a widely used instrument to assess chronic disease care from a patient's perspective. Aim: To adapt the PACIC questionnaire to Chilean Spanish and to evaluate the psychometric properties of the translation. Material and Methods: Descriptive, cross-sectional design with exploratory and confirmatory factor analysis. The PACIC questionnaire was applied, throughout an external client, to 200 users who received health care at the cardiovascular program in a Family Health Center in Concepción, Chile. Results: The confirmatory factor analysis did not show a good adjustment with the proposed structure in the original instrument. An exploratory factor analysis revealed five factors explaining 58\% of total data variability. The distribution of the factor-items of the original questionnaire underwent some modifications, which are explained when analyzing the theoretical construct. A good reliability of the global scale was obtained (Cronbach's $\alpha$ 0.886). Conclusions: The PACIC questionnaire, Chilean version does not replicate the proposed structure of the original questionnaire. Therefore, further research about its validity, incorporating a higher number or diversity of participants is recommended.

(Rev Med Chile 2017; 145: 869-878)

Key words: Chronic Disease; Health Care; Quality Assurance; Validation Studies.

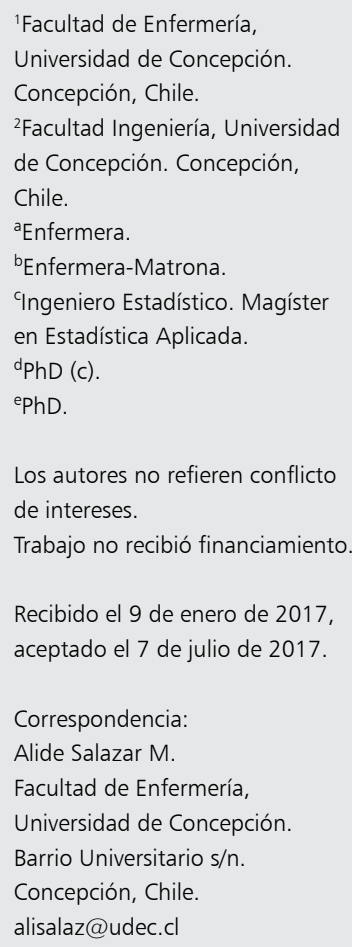

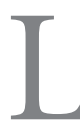

a gestión de la atención de las personas que padecen enfermedades crónicas ha requerido cambios fundamentales ${ }^{1,2}$, debido a la baja efectividad que han demostrado los programas de salud en atención primaria ${ }^{3}$. Es así como en las últimas décadas se han desarrollado nuevos marcos conceptuales, que se articulan a la atención primaria para brindar cuidados basados en evidencia con resultados satisfactorios en la gestión de la atención de pacientes con enfermedades crónicas ${ }^{4-6}$. El modelo de cuidados crónicos de Wagner (MCC) ha demostrado ser un sistema efectivo de entrega de cuidado, orientado en mejorar la calidad de la atención, con énfasis en las interrelaciones del equipo de salud y el usuario, para lograr un equipo competente capaz de organizar la atención, comprender al usuario y otorgar seguridad al paciente y su familia ${ }^{7-9}$.

Es así como, la revisión de literatura revela una amplia evidencia de la utilización del modelo de cuidados crónicos ${ }^{7,8,10-24}$ y de la evaluación de los cuidados proporcionados a los usuarios con en- 
fermedad crónica utilizando como herramienta el cuestionario Patient Assessment of Chronic Illness Care (PACIC) ${ }^{24-36}$. Este instrumento proporciona una evaluación de la atención de enfermedades crónicas desde la perspectiva del paciente $y$, en particular, del apoyo al automanejo ${ }^{24}$.

En la actualidad se dispone de versiones traducidas a distintos idiomas ${ }^{24-35}$, entre las que se encuentran las versiones al español, realizadas por Barceló et al. ${ }^{36}$ y Aragones et $\mathrm{al}^{26}$. Estos últimos realizaron la validación del instrumento al español en hispanoparlantes residentes en Estados Unidos de Norteamérica, reportando niveles satisfactorios de confiabilidad y validez. Sin embargo, considerando las diferencias lingüísticas y culturales entre los distintos contextos en los que fue validado previamente, se estimó necesario realizar una revisión de la formulación de las preguntas contenidas en ambas versiones, concluyendo que no serían adecuadas al lenguaje y contexto chileno, por lo que no sería apropiado su aplicación sin una validación previa.

El objetivo del presente estudio fue describir la adaptación al español hablado en Chile y evaluar las propiedades psicométricas del cuestionario Patient Assessment of Chronic Illness Care. Los resultados de este estudio permitirán disponer de una versión traducida y validada de este instrumento para Chile, que permitirá conocer, desde la perspectiva del usuario, si la atención proporcionada por los equipos de salud es congruente con el MCC.

\section{Sujetos y Métodos}

Estudio descriptivo, trasversal, con análisis factorial confirmatorio y exploratorio.

El instrumento Patient Assessment of Chronic Illness Care (PACIC) está conformado por 20 ítems distribuidos en 5 subescalas: Activación del paciente ( 3 ítems); Diseño de un sistema de entrega de cuidados/Apoyo a la decisión (3 ítems); Diseño de objetivos (5 ítems); Resolución de problemas considerando el contexto del paciente (4 ítems); Seguimiento-Coordinación (5 ítems). Cabe señalar que las 5 subescalas del PACIC no coinciden perfectamente con los 6 componentes del MCC, debido a que algunos aspectos del modelo, tales como sistemas de información clínica y servicios de apoyo, por lo general, no son visibles para los pacientes. No obstante, estos componentes son evaluados a través de la subescala Diseño de un sistema de entrega de cuidados/Apoyo a la decisión. Por su parte el componente del modelo relativo al apoyo al automanejo es evaluado a través de las subescalas Activación del paciente, Diseño de objetivos y Resolución de problemas considerando el contexto del paciente. Asimismo, el cuestionario incorpora una subescala denominada Seguimiento-Coordinación que valora de manera trasversal todos los componentes del MCC. La puntuación de los ítems va desde 1 (nunca) a 5 (siempre) $)^{24}$.

El proceso de traducción y adaptación del PACIC fue realizado siguiendo las normas y las orientaciones de Organización Mundial de la Salud (OMS) ${ }^{37}$. La versión en inglés del PACIC fue traducido al español chileno por 2 traductores de la Universidad de Concepción, de forma independiente. Luego, fue sometido a juicio de expertos, en lo que respecta a la terminología de salud, lo que se resolvió mediante consenso. Posteriormente, la versión en español chileno fue retrotraducida por 1 hablante nativo de inglés y comparada con la versión original. Finalmente, el cuestionario "Evaluación de la atención de enfermedades crónicas para pacientes", versión chilena fue revisado por un lingüista, confirmando una formulación adecuada para el propósito de cada pregunta (Anexo 1).

El cuestionario fue aplicado a 200 usuarios del Programa de Salud Cardiovascular (PSCV) de un centro de salud familiar de Concepción, Chile, quienes fueron reclutados a través de un muestreo por conveniencia. El número de personas se determinó utilizado el criterio de Nunnaly y Bernstein para análisis factorial, considerando de acuerdo a ello 10 sujetos por ítem ${ }^{38}$.

La recolección de datos se realizó durante los meses de noviembre de 2014 a abril de 2015. El cuestionario fue aplicado por encuestadores capacitados a los usuarios del PSCV, quienes fueron informados del objetivo del estudio, solicitándoles su consentimiento informado.

La confiabilidad y consistencia interna del cuestionario total y de las subescalas se efectuó utilizando el coeficiente alpha de Cronbach. Para el análisis factorial confirmatorio (AFC), se utilizó el programa R v. 2.14.0, partiendo de la estructura propuesta por Glasgow et $\mathrm{al}^{24}$. Se seleccionaron índices de bondad de ajuste, siguiendo la propuesta 
de $\mathrm{Hu}$ y Bentler ${ }^{39}$, en que se presentan valores de punto de corte para declarar válido el instrumento: el Standardised Root Mean-Squared Residual (SRMR) $(\leq 0,09)$ en conjunto con valores de punto de corte para Comparative Fit Index (CFI) $(\geq 0,96)$, Root Mean-Squared Error of Approximation (RMSEA) $(\leq 0,06)$ y Non Normed Fit Index (NNFI) $(\geq$ 0,96). Posteriormente, se realizó análisis factorial exploratorio (AFE) considerando el método de componentes principales y rotación varimax. La factibilidad o validación del análisis factorial fue hecha considerando los criterios basados en la medida de adecuación muestral de Kaiser Meyer Olkin (KMO) y en la prueba de esfericidad de Bartlet $^{40}$. Los análisis estadísticos se realizaron utilizando el software SPSS 16.0 para Windows (SPSS Inc, Chicago, Illinois).

El proyecto fue sometido a la consideración del Comité de Ética de la Facultad de Medicina de la Universidad de Concepción. Posteriormente, se solicitó autorización a las autoridades de la Dirección de Salud Municipal, así como a del Centro de Salud Familiar. Se tuvieron en cuenta los requisitos éticos que debe contemplar toda investigación científica, según Ezequiel Emmanuel ${ }^{41}$.

\section{Resultados}

Respecto de las características sociodemográficas de la población estudiada, se encontró que los participantes tenían en promedio 61,58 (DS 12,69) años. Del total de los encuestados, más de la mitad fue de sexo femenino (66\%). Las enfermedades crónicas más prevalentes fueron hipertensión arterial (HTA) (28\%), diabetes mellitus tipo 2 (DM2) (24\%) e HTA y DM2 (21\%). En relación a la situación civil, más de la mitad se encontraba casado (60\%) (Tabla 1).

La Tabla 2 muestra el puntaje promedio y desviación típica de cada uno de los ítems del instrumento. En promedio, los usuarios evaluaron la atención de forma global con un promedio de $(3,06)$. Los mayores puntajes se obtuvieron en los ítems "Me derivaron a ver a un nutricionista u otro profesional de la salud", "Me sentí satisfecho porque el plan de tratamiento estaba bien organizado" y "Me ayudaron a establecer objetivos específicos para mejorar mi alimentación o actividad física”.

El AFC reveló que el instrumento original no presentó un buen ajuste a la estructura original propuesta de cinco factores (RMSEA $=0,086$; $\mathrm{NNFI}=0,762 ;$ SRMR $=0,088, \mathrm{CFI}=0,798)$. Estos resultados dieron origen a la realización de un AFE que permitiese conocer cómo se debería configurar el instrumento considerando los datos recogidos. El AFE identificó 5 factores, bajo el criterio propuesto por Kaiser, autovalor mayor a 1 , que explican $57,96 \%$ de la variabilidad total de los datos $(\mathrm{KMO}=0,885$; Prueba de esfericidad de Bartlett $=0,000)$.

La Tabla 3 muestra la distribución de los ítems-factor, en donde el mayor coeficiente de la fila asociada al ítem indica a qué factor pertenece: Factor 1: compuesto por los ítems 1, 2, 12, 16 y 20. Factor 2: compuesto por los ítems 4, 5, 6, 7, 9 y 15. Factor 3: compuesto por los ítems 3, 8, 11, 13 y 14. Factor 4: compuesto por los ítems 10 y 17. Factor 5: compuesto por los ítems 18 y 19.

La Tabla 4 muestra los puntajes promedio del cuestionario de forma global, así como también el puntaje promedio de cada uno de los factores obtenidos. Del mismo modo, esta tabla muestra la consistencia interna del instrumento global, así como de cada uno de los factores en donde se observa que a nivel global el coeficiente alpha de Cronbach es de 0,86 y que para cuatro de los cinco

\section{Tabla 1. Características sociodemográficas de participantes del estudio $(n=200)$}

\begin{tabular}{|l|l|}
\hline Variable & \\
Edad (Media, DE) & $61,58 \quad 12,69$ \\
Sexo (\%) & \\
$\quad$ Femenino & $66 \%$ \\
Masculino & $34 \%$ \\
Estado civil (\%) & \\
Soltero (a) & $13 \%$ \\
Casado(a) & $62,5 \%$ \\
Divorciado(a) & $6,5 \%$ \\
Viudo(a) & $18 \%$ \\
Enfermedades crónicas (\%) & \\
Hipertensión (HTA) & $28 \%$ \\
Diabetes mellitus tipo 2 (DM2) & $24 \%$ \\
HTA y DM2 & $21 \%$ \\
\hline Años de tratamiento (Media, DE) & $9,37 \quad 7,12$ \\
\hline
\end{tabular}

DE: Desviación estándar; \%: Porcentaje. 
Tabla 2. Puntaje promedio por ítems “Evaluación de la atención de enfermedades crónicas para pacientes"

\begin{tabular}{|c|c|c|}
\hline Ítem & Media & DE \\
\hline 1. Me preguntaron sobre mis ideas a la hora de diseñar un plan de tratamiento & 2,5 & 1,8 \\
\hline 2. Me dieron opciones de tratamiento para que las considerara & 2,5 & 1,9 \\
\hline 3. Me preguntaron si tenía problemas con mis medicamentos o sus efectos & 3,7 & 1,7 \\
\hline 4. Me entregaron una lista por escrito con las cosas que debería hacer para mejorar mi salud & 3,1 & 1,9 \\
\hline 5. Me sentí satisfecho porque el plan de tratamiento estaba bien organizado & 4 & 1,4 \\
\hline 6. Me mostraron cómo mis acciones para cuidarme influyeron en mi estado de salud & 3,4 & 1,8 \\
\hline 7. Me pidieron que hablara sobre mis metas respecto al cuidado de mi enfermedad & 2,3 & 1,8 \\
\hline 8. Me ayudaron a establecer objetivos específicos para mejorar mi alimentación o actividad física & 4 & 1,6 \\
\hline 9. Me entregaron una copia de mi plan de tratamiento & 2,7 & 1,9 \\
\hline $\begin{array}{l}\text { 10. Me alentaron a asistir a una clase o grupo en particular para que pudiera convivir mejor con mi } \\
\text { enfermedad crónica }\end{array}$ & 2,8 & 1,9 \\
\hline $\begin{array}{l}\text { 11. Me hicieron preguntas, ya sea directas o en forma de encuesta, sobre mis hábitos relacionados } \\
\text { con la salud }\end{array}$ & 3,2 & 1,8 \\
\hline $\begin{array}{l}\text { 12. Estoy seguro/a que mi médico o la enfermera(o) tomaban en cuenta mis valores, creencias y } \\
\text { tradiciones cuando me recomendaron algún tratamiento }\end{array}$ & 2,9 & 1,9 \\
\hline 13. Me ayudaron a para diseñar un plan de tratamiento que pudiera llevar a cabo en mi vida diaria & 3,8 & 1,7 \\
\hline $\begin{array}{l}\text { 14. Me ayudaron a para planificar con anticipación, de modo que pudiera cuidar mi salud incluso en } \\
\text { tiempos difíciles }\end{array}$ & 2,8 & 1,8 \\
\hline 15. Me preguntaron cómo mi enfermedad crónica ha afectado mi vida & 2,5 & 1,8 \\
\hline 16. Me contactaron después de una consulta para saber cómo estaba & 2,2 & 1,8 \\
\hline 17. Me alentaron a participar en programas dentro de la comunidad que me podrían ayudar & 2,9 & 2,1 \\
\hline 18. Me derivaron a ver a un nutricionista u otro profesional de la salud & 4,3 & 1,5 \\
\hline $\begin{array}{l}\text { 19. Me hablaron sobre los beneficios para mi tratamiento que tuvo la consulta con otros especialistas, } \\
\text { como un oftalmólogo o un }\end{array}$ & 2,8 & 1,9 \\
\hline 20. Me preguntaron cómo me estaba yendo las consultas con otros médicos & 2,3 & 1,8 \\
\hline
\end{tabular}

Puntaje de 1 a 5 (1: nunca; 2: pocas veces; 3: a veces; 4 la mayoría de las veces; 5: siempre).

factores definidos el coeficiente alpha de Cronbach es superior a 0,6 .

La Tabla 5 presenta la comparación entre el instrumento original y la agrupación de ítems a partir del análisis factorial exploratorio realizado.

\section{Discusión}

Con el fin de evaluar el comportamiento del instrumento y la forma en que se agrupan los ítems dentro de cada una de las dimensiones, se realizó un AFC que buscó determinar en qué medida el modelo teórico de cinco factores se ajusta a los datos muestrales, arrojando que el instrumento original no presentó un buen ajuste. Estos resultados dieron origen a la realización de un AFE que permitiese conocer cómo se debería configurar el instrumento considerando los datos recogidos.

El AFE detectó cinco factores, identificándose ítems que se distribuyeron con algunas modificaciones respecto de la conformación original del PACIC. Para explicar la composición de los factores es necesario considerar que cada ítem se diseñó para medir el constructo teórico, es por esto que, para interpretar mejor la relación de ítemfactor, se efectúo rotación varimax obteniéndose correlaciones con aportes relevantes (0,7 y más), 
Evaluación de atención de enfermedades crónicas para pacientes - M. E. Lagos et al

Tabla 3. Análisis factorial con rotación varimax

\begin{tabular}{|c|c|c|c|c|c|}
\hline \multirow[b]{2}{*}{ Ítems } & \multicolumn{5}{|c|}{ Factores } \\
\hline & 1 & 2 & 3 & 4 & 5 \\
\hline Me preguntaron sobre mis ideas a la hora de diseñar un plan de tratamiento & 0,795 & 0,053 & 0,165 & 0,056 & 0,103 \\
\hline Me dieron opciones de tratamiento para que las considerara & 0,721 & 0,226 & 0,211 & $-0,1$ & 0,097 \\
\hline Me preguntaron si tenía problemas con mis medicamentos o sus efectos & 0,199 & $-0,02$ & $-0,62$ & 0,066 & $-0,21$ \\
\hline $\begin{array}{l}\text { Me entregaron una lista por escrito con las cosas que debería hacer para } \\
\text { mejorar mi salud }\end{array}$ & $-0,15$ & 0,585 & 0,378 & 0,183 & 0,168 \\
\hline Me sentí satisfecho, porque el plan de tratamiento estaba bien organizado & 0,02 & 0,413 & 0,346 & 0,016 & 0,358 \\
\hline $\begin{array}{l}\text { Me mostraron cómo mis acciones para cuidarme influyeron en mi estado } \\
\text { de salud }\end{array}$ & 0,181 & 0,701 & 0,136 & $-0,06$ & 0,177 \\
\hline $\begin{array}{l}\text { Me pidieron que hablara sobre mis metas respecto al cuidado de mi enfer- } \\
\text { medad }\end{array}$ & 0,464 & $-0,601$ & 0,113 & 0,069 & $-0,17$ \\
\hline $\begin{array}{l}\text { Me ayudaron a establecer objetivos específicos para mejorar mi alimentación } \\
\text { o actividad física }\end{array}$ & 0,055 & 0,361 & 0,411 & 0,293 & 0,261 \\
\hline Me entregaron una copia de mi plan de tratamiento & 0,362 & 0,478 & 0,15 & 0,185 & 0,116 \\
\hline $\begin{array}{l}\text { Me alentaron a asistir a una clase o grupo en particular para que pudiera } \\
\text { convivir mejor con mi enfermedad crónica }\end{array}$ & 0,086 & 0 & 0,142 & 0,867 & 0,093 \\
\hline $\begin{array}{l}\text { Me hicieron preguntas, ya sea directas o en forma de encuesta, sobre mis } \\
\text { hábitos relacionados con la salud }\end{array}$ & 0,189 & 0,256 & 0,532 & 0,271 & 0,184 \\
\hline $\begin{array}{l}\text { Estoy seguro/a que mi médico o la enfermera(o) tomaban en cuenta mis } \\
\text { valores, creencias y tradiciones cuando me }\end{array}$ & 0,534 & 0,244 & 0,508 & 0,164 & 0,108 \\
\hline $\begin{array}{l}\text { Me ayudaron a diseñar un plan de tratamiento que pudiera llevar a cabo en } \\
\text { mi vida diaria }\end{array}$ & 0,191 & 0,196 & 0,728 & 0,042 & 0,228 \\
\hline $\begin{array}{l}\text { Me ayudaron a planificar con anticipación, de modo que pudiera cuidar mi } \\
\text { salud incluso en tiempos difíciles }\end{array}$ & 0,293 & 0,336 & 0,451 & 0,044 & $-0,21$ \\
\hline Me preguntaron cómo mi enfermedad crónica ha afectado mi vida & 0,317 & 0,616 & 0,087 & 0,126 & 0,054 \\
\hline Me contactaron después de una consulta para saber cómo estaba & 0,56 & 0,475 & 0,004 & 0,288 & $-0,16$ \\
\hline $\begin{array}{l}\text { Me alentaron a participar en programas dentro de la comunidad que me } \\
\text { podrían ayudar }\end{array}$ & 0,059 & 0,176 & 0,1 & 0,847 & 0,088 \\
\hline Me derivaron a un nutricionista $u$ otro profesional de la salud & 0,138 & 0,036 & $-0,04$ & 0,098 & 0,835 \\
\hline $\begin{array}{l}\text { Me hablaron sobre los beneficios para mi tratamiento que tuvo la consulta } \\
\text { con otros especialistas }\end{array}$ & 0,173 & 0,342 & 0,148 & 0,303 & 0,454 \\
\hline Me preguntaron cómo me estaba yendo con las consultas con otros médicos & 0,598 & 0,172 & 0,248 & 0,183 & 0,306 \\
\hline
\end{tabular}

Tabla 4. Puntaje promedio y confiabilidad “Evaluación de la atención de enfermedades crónicas para pacientes"

\begin{tabular}{|lrcc|}
\hline Escala & $\begin{array}{c}\text { n de } \\
\text { ítems }\end{array}$ & $\begin{array}{c}\text { Media } \\
\text { (DE) }\end{array}$ & $\begin{array}{c}\text { Consistencia interna } \\
\text { ( } \boldsymbol{\alpha} \text { de Cronbach) }\end{array}$ \\
\hline Total & 20 & $3,06(0,64)$ & 0,886 \\
\hline Factor 1 Activación del paciente & 5 & $2,91(0,7)$ & 0,617 \\
\hline Factor 2 Diseño de un sistema de entrega de cuidados/Apoyo en la decisión & 6 & $3,51(0,46)$ & 0,749 \\
\hline Factor 3 Diseño de objetivos & 5 & $2,94(0,74)$ & 0,686 \\
\hline Factor 4 Resolución de problemas considerando contexto del paciente & 2 & $3,03(0,5)$ & 0,777 \\
\hline Factor 5 Seguimiento-Coordinación & 2 & $2,91(0,82)$ & 0,48 \\
\hline
\end{tabular}




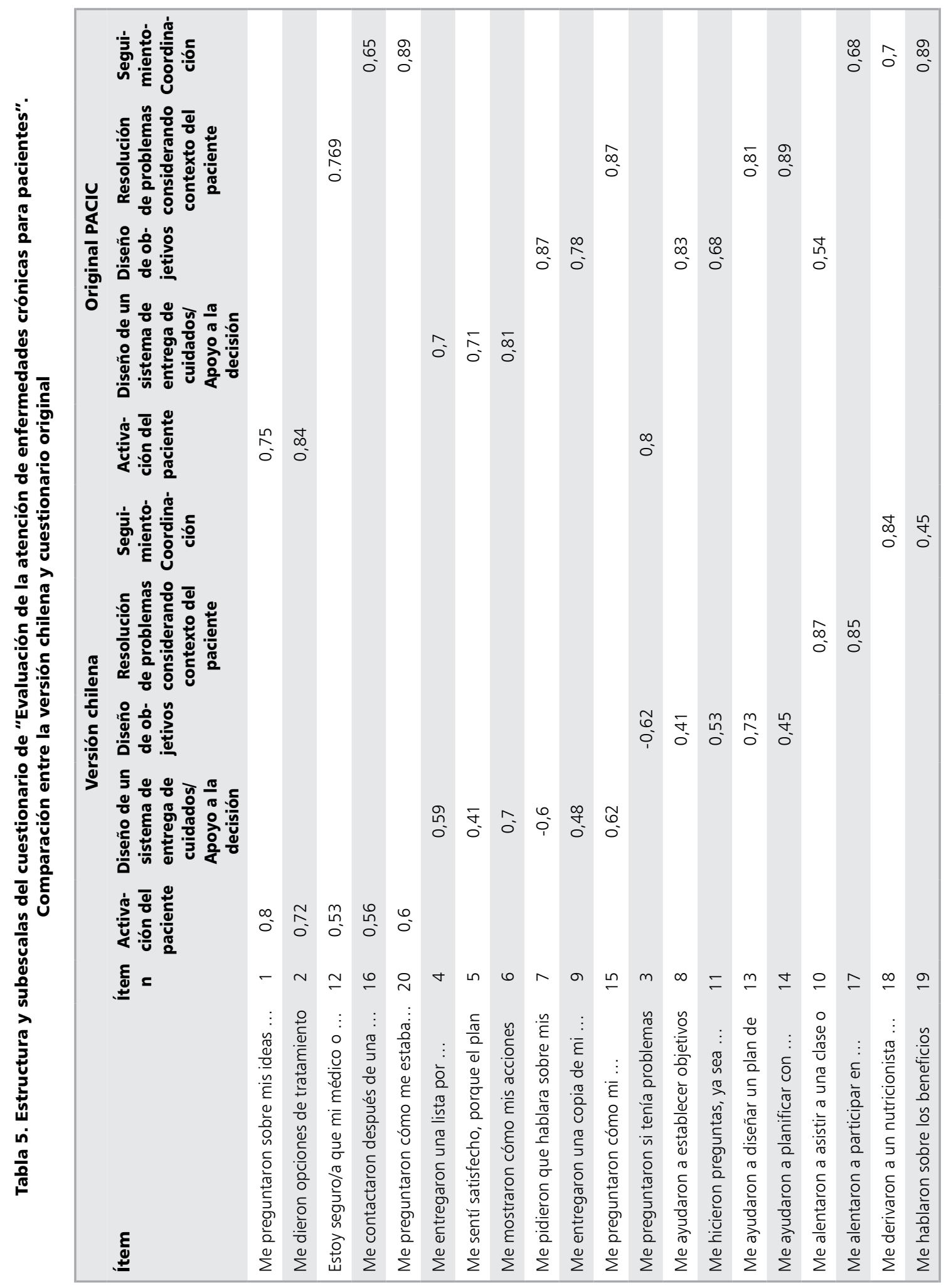


significativas (desde 0,5 y 0,7 ), aportes mínimos $(0,3$ hasta 0,5$)$ y no significativo $(0 \text { hasta } 0,3)^{42}$.

Es así como el factor 1, denominado "Activación del paciente" se conformó por 5 ítems, a diferencia de la subescala original $(1,2$ y 3$)$, manteniendo 2 ítems de "Activación del paciente" e incorporando 1 ítem de "Resolución de problemas" y 2 ítems de "Seguimiento-Coordinación". Lo anterior puede explicarse debido a que los ítems 1,2 y 12 , que conformaron este factor, están directamente relacionados con el constructo teórico original, el cual mide directamente los rasgos del componente del MCC "apoyo al automanejo", a través de las subescalas "Activación del paciente" y "Resolución de problemas", representando en su conjunto las acciones que efectúa el equipo de salud para considerar al usuario participe de la toma de decisiones en su salud. Sin embargo, incorporó 2 ítems de "Seguimiento-Coordinación”, cuya característica es medir las acciones efectuadas entre el usuario y el equipo para manejar de mejor forma la condición de salud, aportando de manera trasversal a todos los elementos del MCC.

El factor 2 "Diseño de un sistema de entrega de cuidados/Apoyo en la decisión”, también sufrió modificaciones, ya que se correlacionó con 6 ítems. Este factor mantuvo los 3 ítems de "Diseño de un sistema de entrega de cuidados/Apoyo en la decisión" (4, 5 y 6), sin embargo, también se correlacionó de forma significativa el ítem $15 \mathrm{co-}$ rrespondiente a "Resolución de problemas" y los ítems 7 y 9 de la subescala "Diseño de objetivos". Respecto de este último, la correlación puede atribuirse a que en el contexto chileno el usuario percibe esta actividad como parte de las acciones efectuadas para disponer y organizar la atención.

El factor 3, "Diseño de objetivos", se conformó por 5 ítems, manteniendo 2 ítems ( 8 y 11) de la subescala original e incorporando 1 ítem $^{3}$ de "activación del paciente" y 2 ítems (13 y 14) de "Resolución de problemas considerando contexto del paciente". Lo anterior puede explicarse debido a que los ítems que conformaron este factor están relacionados con la construcción teórica que mide directamente los rasgos del componente del MCC "apoyo al automanejo", a través de las subescala "Activación del paciente", "Resolución de problemas" y "Diseño de objetivos" y que en su conjunto revelan las acciones efectuadas por el equipo de salud para desarrollar un plan de tratamiento adecuado con el usuario. Cabe mencionar que los ítems 8 y 14 se correlacionaron otorgando un aporte mínimo, lo que podría sugerir su eliminación en futuros análisis.

Respecto del factor 4, "Resolución de problemas considerando contexto del paciente", sólo incorporó 2 ítems, a diferencia de la subescala original que estaba compuesta por 4 ítems (12, 13,14 y 15), manteniendo 1 ítem de "Resolución de problemas considerando contexto del paciente" y agregando 1 ítem de "Diseño de objetivos". Lo anterior se puede deber a que ambos ítems describen al componente que dio origen a su construcción "apoyo al automanejo", en el que se consideran el entorno social y las posibles barreras para dar cumplimiento a su plan terapéutico. En este sentido, la fuerza con que ambas variables se correlacionan con el factor fueron relevantes para su incorporación.

Por último, el factor 5, "Seguimiento-Coordinación", se formó por 2 de los ítems originales 18 y 19. Por otra parte, para este factor podrían ser redundante los ítems 16, 17 y 20, que originalmente lo componían, ya que ellos caracterizan de mejor forma a las subescalas "Activación del paciente" y "Resolución de problemas considerando contexto del paciente", respectivamente.

Como es posible observar, los resultados del análisis factorial muestran un apoyo parcial a la conformación de las cinco subescalas definidas originalmente en el PACIC. Cabe señalar que estudios publicados han mostrado resultados discordantes en la validez factorial del instrumento. Es así como Gigiu et al. ${ }^{31}$ encontraron 1 a 2 factores, señalando que su estructura correspondería más bien a un constructo unidimensional, similar a lo reportado por Aung et al..$^{34}$, quienes identificaron una estructura de un factor. Por otra parte, Fan et $\mathrm{al}^{35}$. hallaron un modelo de 4 y no de 5 factores. Por lo tanto, se recomienda más investigación sobre su validez, que incorpore un mayor número de participantes y en otros grupos de pacientes con enfermedades crónicas, a fin de revisar la estructura de subescalas.

Respecto de la confiabilidad del cuestionario, se obtuvo una buena confiabilidad global ( $\alpha$ de Cronbach 0,886 ), encontrándose valores mayores a 0,6 para cuatro de los cinco factores, lo cual puede considerarse aceptable para escalas breves ${ }^{40,42}$. Estos resultados son similares a los reportados por Aragones et al. ${ }^{26}$ al estudiar las propiedades psicométricas de la validación al español en una 
muestra de hispanoparlantes residentes en Estados Unidos de Norteamérica.

En conclusión, el cuestionario "Evaluación de la atención de enfermedades crónicas para paciente (PACIC)" ha demostrado potencial utilidad para evaluar la atención recibida por el usuario con enfermedad crónica ${ }^{22}$, sin embargo, la versión chilena no logra replicar la estructura propuesta en el cuestionario original. Por lo tanto, se recomienda más investigación sobre su validez, que incorpore un mayor número y diversidad de participantes, ya que este cuestionario puede orientar al sistema de salud a proveer una atención eficiente, efectiva y equitativa en personas con factores de riesgo y con más de una enfermedad crónica (multimorbilidad) ${ }^{43}$.

Es importante señalar que este estudio involucra varias limitaciones. En primer lugar, este estudio sólo reclutó personas que percibían atención de un establecimiento perteneciente al sistema público de Chile, lo que pudo influir en las respuestas considerando como variable el entorno. Lo anterior responde a que las personas que reciben atención en este ámbito público pueden evaluar su cuidado de forma diferente de aquellas que reciben atención en el ámbito privado. En segundo lugar, no se examinó la fiabilidad test-retest del cuestionario ni la validez concurrente (o externa) de esta adaptación, por lo que se considera pertinente continuar investigando para confirmar los resultados de este estudio, mejorar la composición de los componentes encontrados e involucrar a otras regiones geográficas de Chile.

\section{Referencias}

1. World Health Organization. Preventing chronic diseases: a vital investment: WHO global report [Internet]. Canadá. 2005 [citado el 25 de marzo de 2015]. Disponible en: http://www.who.int/chp/chronic_disease_report/ full_report.pdf

2. Organización Panamericana de Salud. Cuidado Innovador para las condiciones crónicas: agenda para el cambio. Informe global. Atención de Salud para las Condiciones Crónicas. Gestión de Enfermedades no Transmisibles. Enfermedades no Transmisibles y Salud Mental [Internet]. 2003 [citado el 25 de marzo de 2015]. Disponible en: http://www.who.int/chp/knowledge/ publications/iccc_spanish.pdf

3. Starfield B. Point: The Changing Nature of Disease:
Implications for Health Services. Med Care [Internet]. 2011 [citado el 10 de mayo de 2017]; 49 (11): 971-2. Disponible en: http://www.jstor.org/stable/23053785.

4. Wagner E. Counterpoint: chronic illness and primary care. Med Care [Internet]. 2011 Nov [citado el 10 de mayo de 2017]; 49 (11): 973-5. Disponible en: http:// www.jstor.org/stable/23053785.

5. Organización Mundial de la Salud. Preparación de los profesionales de la atención de salud para el siglo XXI: el reto de las enfermedades crónicas. Enfermedades No Transmisibles y Salud Mental Enfermedades Crónicas y Promoción de la Salud. Ginebra [Internet]. 2005 [citado el 10 de abril de 2015]. Disponible en: http://apps. who.int/iris/bitstream/10665/43236/1/9243562800_spa. pdf

6. Ministerio de Salud de Chile. Estrategia nacional de salud para el cumplimiento de los objetivos sanitarios para la década 2011-2020: elige vivir sano [Internet]. 2010 Abr [citado el 25 de abril de 2015]. Disponible en: http://www.minsal.cl/portal/url/item/c4034eddbc96ca6de0400101640159b8.pdf

7. Wagner EH, Austin B, Davis C, Hindmarsh M, Schaefer J, Bonomi A. Improving Chronic Illness Care: Translating Evidence Into Action. Health Aff [Internet]. 2001 Nov [citado el 3 de junio de 2015]; 20 (6): 64-78. Disponible en: http://content.healthaffairs.org/content $/ 20 / 6 / 64$.

8. Bodenheimer T, Wagner EH, Grumbach K. Improving Primary Care for Patients with Chronic Illness. JAMA [Internet]. 2002 [citado el 9 de junio de 2015]; 288: (14): 1775-9. Disponible en: https://www.ncbi.nlm.nih.gov/ pubmed/12365965.

9. Ministerio de Salud de Chile. Modelo de atención para personas con enfermedades crónicas. Directrices para la Implementación [Internet]. 2015 Jun [citado el 27 de mayo de 2015]. Disponible en: http://www.enfermeriaaps.com/portal/modelo-de-atencion-para-personas-con-en

10. Bowen JL, Wagner EH, Stevens DP. Chronic Care and Education. J Gen Intern Med [Internet]. 2010 Sep. [citado el 7 de julio de 2015]; 25 Suppl 4: 569. Disponible en: https://www.ncbi.nlm.nih.gov/pubmed/20737229.

11. Johnson JK, Woods DM, Stevens DP, Bowen JL Provost LLP, Sixta CS, et al. Joy and Challenges in Improving Chronic Illness Care: Capturing Daily Experiences of Academic Primary Care Teams. J Gen Intern Med [Internet]. 2010 Sep [citado el 9 de julio de 2015]; 25 Suppl 4: 581-5. Disponible en: https://www.ncbi.nlm.nih.gov/ pubmed/20737233.

12. Renders CM, Valk GD, Griffin GJ, Wagner EH. Eijk Van JT, Assendelft WJ. Interventions to Improve the 
Management of Diabetes in Primary Care, Outpatient, and Community Settings: a systematic review. Diabetes care [Internet]. 2001 Oct [citado el 9 de julio de 2015]; 24: (10): 1821-33. Disponible en: https://www.ncbi.nlm. nih.gov/pubmed/11574449.

13. Solberg LF, Crain L, Sperl-Hillen J, Hroscikoski M, Engebretson K, O'Connor P. Care Quality and Implementation of the Chronic Care Model: A Quantitative Study. Ann Fam Med [Internet]. 2006 Jul [citado el 9 de agosto de 2015]; 4: (4): 310-6. Disponible en: http:// www.annfammed.org/content/4/4/310.full.

14. Leykum LK, Palmer R, Lanham H, Jordan M, McDaniel RR, Noël PH, et al. Reciprocal learning and chronic care model implementation in primary care: results from a new scale of learning in primary care Leykum et al. Health Services Research. [Internet]. 2011 Feb [citado el 10 de septiembre de 2015]; 11: (44). Disponible en: https://bmchealthservres.biomedcentral.com/articles/10.1186/1472-6963-11-44.

15. Tsai AC, Morton SC, Mangione CM, Keeler EB. A Meta-Analysis of Interventions to Improve Care for Chronic Illnesses. Am J Manag Care [Internet]. 2005 Ago [citado el 11 de septiembre de 2015]; 11: (8): 478-88. Disponible en: https://www.ncbi.nlm.nih.gov/ pubmed/16095434.

16. Bonomi AE, Wagner EH, Glasgow RE, Vonkorff M. Assessment of Chronic Illness Care (ACIC): A Practical Tool to Measure Quality Improvement HSR. Health Serv Res [Internet]. 2002 Jun [citado el 13 de septiembre de 2015]; 37 (3): 791-820. Disponible en: https://www. ncbi.nlm.nih.gov/pubmed/12132606.

17. Cramm JM, Strating MM, Tsiachristas A, Nieboer AP. Development and validation of a short version of the Assessment of Chronic Illness Care (ACIC) in Dutch Disease Management Programs. Health Qual Life Outcomes [Internet]. $2011 \mathrm{Jul}$ [citado el 12 de octubre de 2015]; 9:49. Disponible en: https://www.ncbi.nlm.nih. gov/pubmed/21726439.

18. Steinhaeuser J, Goetz K, Ose D, Glassen K, Natanzon I, Campbell S, et al. Applicability of the assessment of chronic illness care (ACIC) instrument in Germany resulting in a new questionnaire: questionnaire of chronic illness care in primary care. BMC Health Services Research [Internet]. 2011Jul [citado el 12 de octubre de 2015]; 7 (11): 164. Disponible en: http://www.biomedcentral.com/1472-6963/11/164.

19. Steurer-Stey C, Frei A, Schmid-Mohler G, Malcolm-Kohler S, Zoller M, Rosemann T. Assessment of Chronic Illness Care with the German version of the ACIC in different primary care settings in Switzerland. Health Qual Life Outcomes [Internet]. 2010 Oct [citado el 5 de noviembre de 2015]; 8: 122. Disponible en: http://www. hqlo.com/content/8/1/122.

20. Lemmens KM, Nieboer AP, Van Schayck CP, Asin JD, Huijsman R. A model to evaluate quality and effectiveness of disease management. Qual Saf Health Care [Internet]. 2008 Dic [citado el 6 de diciembre de 2015]; 17 (6): 447-53. Disponible en: https://www.ncbi.nlm. nih.gov/pubmed/19064661.

21. Whitlock E, Orleans T, Pender N, Allan J. Evaluating Primary Care Behavioral Counseling Interventions An Evidence-Based Approach. Am J Prev Med [Internet]. 2002 May [citado el 8 de enero de 2016]; 22(4): 267-84. Disponible en: https://www.ncbi.nlm.nih.gov/pubmed/11988383.

22. Lorig K, Ritter PL, Villa F, Piette JD. Spanish diabetes self-management with and without automated telephone reinforcement: two randomized trials. Diabetes Care [Internet] 2008 Mar [citado el 12 de enero de 2016]; 31(3): 408-14. Disponible en: https:// doi.org/10.2337/dc07http://care.diabetesjournals.org/ content/31/3/408.

23. Parchman ML, Pugh JA, Wang CP, Romero RL. Glucose control, self-care behaviors, and the presence of the chronic care model in primary care clinics. Diabetes Care [Internet]. 2007 Nov [citado el 15 de enero de 2016] ; 30 (11):2849-2554. Disponible en:

24. Glasgow RE, Wagner EH, Schaefer J, Mahoney LD, Reid RJ, Greene SM. Development and validation of the Patient assessment of Chronic Illness Care (PACIC) Med Care [Internet]. 2005 May [citado el 20 de abril de 2017]; 43: 436-44. Disponible en: https://www.ncbi.nlm. nih.gov/pubmed/15838407.

25. Glasgow RE, Whitesides H, Nelson CC, King DK. Use of the Patient Assessment of Chronic Illness Care (PACIC) with diabetic patients: relationship to patient characteristics, receipt of care, and self-management. Diabetes Care [Internet]. 2005 Nov [citado el 20 de marzo de 2016]; 28(11):2655-2661. Disponible en: http://care. diabetesjournals.org/content/28/11/2655.

26. Aragones A, Schaefer E, Stevens D, Gourevitch M, Shah N, Glasgow R. Validation of the Spanish Translation of the Patient Assessment of Chronic Illness Care (PACIC). Survey. Prev Chronic Dis [Internet]. 2008 Oct [citado el 30 de marzo de 2016]; 5 (4): 113. Disponible en: https://www.ncbi.nlm.nih.gov/pmc/articles/ PMC2578783/

27. Rick J, Rowe K, Hann M, Sibbald B, Reeves D, Roland $\mathrm{M}$, et al. Psychometric properties of the patient assessment of chronic illness care measure: acceptability, reliability and validity in United Kingdom patients with long-term conditions. BCM Health Serv Res [Internet]. 
2012 Ago [citado 10 de abril de 2016]; 12: 293 Disponible en: http://bmchealthservres.biomedcentral.com/ articles/10.1186/1472-6963-12-293.

28. Szecsenyi J, Rosemann T, Joos S, Peters-Klimm F, Miksch A. German diabetes disease management programs are appropriate for restructuring care according to the chronic care model: an evaluation with the patient assessment of chronic illness care instrument. Diabetes Care [Internet]. 2008 Jun [citado el 10 de abril de 2016]; 31 (6): 1150-4. Disponible en: http://care.diabetesjournals.org/content/31/6/1150.

29. Vrijhoef HJ, Berbee R, Wagner EH, Steuten LM. Quality of integrated chronic care measured by patient survey: identification, selection and application of most appropriate instruments. Health Expect [Internet]. 2009 Dic [citado el 25 de mayo de 2016]; 12 (4): 417-29. Disponible en: https://www.ncbi.nlm.nih.gov/pubmed/19709315.

30. Cramm JM, Nieboer AP. Factorial validation of the patient assessment of chronic illness care (PACIC) and PACIC short version (PACIC-S) among cardiovascular disease patients in the Netherlands. Health Qual Life Outcomes [Internet]. 2012 Ago [citado el 10 de mayo de 2016]; 10: 104. Disponible en: https://hqlo.biomedcentral.com/articles/10.1186/1477-7525-10-104.

31. Gugiu C, Coryn CL, Applegate B. Structure and measurement properties of the Patient Assessment of Chronic Illness Care instrument. J Eval Clin Pract. [Internet]. 2010 Jun [citado el 20 de julio de 2016]; 16 (3): 509-16. Disponible en: https://www.ncbi.nlm.nih.gov/pubmed/20210824.

32. Wensing M, Lieshout J, Jung H, Hermsen J, Rosemann T. The Patients Assessment Chronic Illness Care (PACIC) questionnaire in The Netherlands: a validation study in rural general practice. Health Services Research [Internet]. 2008 Sep [citado el 20 de agosto de 2016]; 8: 182. Disponible en: http://bmchealthservres.biomedcentral.com/articles/10.1186/1472-6963-8-182.

33. Krucien N, Vaillant M, Pelletier-Fleury N. Adaptation and validation of the patient assessment of chronic illness care in the French context. BMC Health Services Research [Internet]. 2014 Jun [citado el 30 de agosto de 2016]; 14: 269 https://www.ncbi.nlm.nih.gov/pmc/ articles/PMC4094668/pdf/1472-6963-14-269.pdf

34. Aung E, Ostini R, Dower J, Donald M, Coll JR, Williams
GM, et al. Patient Assessment of Chronic Illness Care (PACIC) in Type 2 Diabetes: A Longitudinal Study. Eval Health Prof [Internet]. 2016 Jun [citado el 18 de diciembre de 2016]; 39 (2): 185-203. Disponible en: https://www.ncbi.nlm.nih.gov/pubmed/25380699.

35. Fan J, McCoy RG, Ziegenfuss JY, Smith SA, Borah BJ, Deming JR, et al. Evaluating the structure of the Patient Assessment of Chronic Illness Care (PACIC) survey from the patient's perspective. Ann Behav Med [Internet]. 2015 Feb [citado el 18 de diciembre de 2016]; 49 (1): 104-11. Disponible en: https://www.ncbi.nlm.nih. gov/pubmed/25236671.

36. Improving Chronic Illness Care. Resource library. Survey Instruments [Internet]. [citado el 11 de noviembre de 2016]. Disponible en: http://www.improvingchroniccare.org/index.php?p=Survey_Instruments\&s=165.

37. World Health Organization. Process of translation and adaptation of instruments. Research tools. Management of substance abuse [Internet]. [citado el 10 de septiembre de 2016] Disponible en: http://www.who. int/substance_abuse/research_tools/translation/en/

38. Nunnally JC, Bernstein IJ. Teoría psicométrica. 3ra ed. México. Editorial Latinoamericana. 1995.

39. Hu L, Bentler PM. Cutoff criteria for fit indices in covariance structure analysis: conventional criteria versus new alternatives. Struct Equ Modeling 1999; 6: 1-55.

40. Oviedo C, Campo A. Aproximación al uso del coeficiente alfa de Cronbach. Revista Colombiana de Psiquiatría [Internet]. 2005 [citado el 16 de diciembre de 2016]; XXXIV: (4): 572-80. Disponible en: http://www.redalyc. org/articulo.oa?id=80634409.

41. Rodríguez E. Comités de Evaluación Ética y Científica para la Investigación en Seres Humanos y las Pautas CIOMS 2002. Acta bioeth 2004; 10 (1): 37-48.

42. Streiner D. Starting at the Beginning: An Introduction to Coefficient Alpha and Internal. Journal of personality assessment [Internet]. 2010 Jun [citado el 16 de diciembre de 2016]; 80 (1): 99-103. Disponible en: http:// dx.doi.org/10.1207/S15327752JPA8001_18.

43. Smith SM, Wallace E, O'Dowd T, Fortin M. Interventions for improving outcomes in patients with multimorbidity in primary care and community settings. Cochrane Database Syst Rev [Internet]. 2016 Mar [citado el 20 de abril de 2017]; 14; 3. Disponible en: https://www. ncbi.nlm.nih.gov/pubmed/26976529. 